\title{
Helium abundance and ionization structure in the Orion nebula: radio recombination lines observations ${ }^{\star}$
}

\author{
S. Poppi ${ }^{1}$, A. P. Tsivilev ${ }^{2,3}$, S. Cortiglioni ${ }^{4}$, G. G. C. Palumbo ${ }^{5}$, and R. L. Sorochenko ${ }^{2}$ \\ 1 INAF - Osservatorio Astronomico di Cagliari, Loc. Poggio dei Pini, Str. 54, 09012 Capoterra (CA), Italy \\ e-mail: spoppi@ca.astro.it \\ 2 P.N Lebedev Physical Institute, Academy of Sciences of Russia, Moscow, Russia \\ Isaac Newton Institute of Chile, Pushchino Branch, Pushchino, 142290 Russia \\ 4 INAF - IASF Sezione di Bologna, via P. Gobetti 101, 40129 Bologna, Italy \\ 5 Università degli Studi, Dipartimento di Astronomia, 40129 Bologna, Italy
}

Received 5 November 2004 / Accepted 27 November 2006

\section{ABSTRACT}

\begin{abstract}
Results of the Ori A HII region mapping based on hydrogen $(\mathrm{H})$, helium $(\mathrm{He})$ and carbon $(\mathrm{C})$ Radio Recombination lines (RRL) are presented. Observations were made with the same angular resolution ( $2^{\prime}$ ) using the $32 \mathrm{~m}$ VLBI dish of Medicina (Italy, $22.4 \mathrm{GHz}$ ) and the Pushchino RT-22 dish (Russia, $36.5 \mathrm{GHz}$ ). The behaviour of the ionized helium abundance, $y^{+}$, with distance from the center shows that the $\mathrm{He}^{+}$zone size is smaller than that of $\mathrm{H}^{+}$. Such a behaviour is different for the core and for the envelope, as well as for different directions from the center. The helium abundance, $N(\mathrm{He}) / N(\mathrm{H})=10.0( \pm 0.8) \%$, is measured. Derived line radial velocities, their widths and $y^{+}$data support the well-known "blister-type" structure of this HII region. LTE electron temperatures (7800-9600 K) are also measured.
\end{abstract}

Key words. ISM: abundances - ISM: HII regions - radio lines: ISM - ISM: individual objects: Orion Nebula

\section{Introduction}

The relative helium abundance, $y=N(\mathrm{He}) / N(\mathrm{H})$, is one of the main parameters of Cosmology and Astrophysics related to primordial nucleosynthesis and galaxy evolution (Zel'dovich \& Novikov 1975; Walker et al. 1991) that can reliably be measured by means of Radio Recombination Line (RRL) observations in HII regions (Churchwell et al. 1974; Schmid-Burgk 1981; Sorochenko \& Tsivilev 1996). Such observations, however, as for similar observations at optical and IR wavelengths, measure the ratio of the ionized component, $y^{+}=N\left(\mathrm{He}^{+}\right) / N\left(\mathrm{H}^{+}\right)$. The problem of deriving the correct value of $y$ from the measured $y^{+}$ therefore remains unresolved.

The measured $y^{+}$values greatly differ from one another and, in galactic HII regions, span from $\sim 3 \%$ to $\sim 12 \%$ (Shaver et al. 1983). Even though this scatter can be partially explained by observational difficulties (Sorochenko \& Tsivilev 1996; Gordon \& Sorochenko 2002), the lack of a deep knowledge of the HII region ionization structures still represents the main problem. Most determinations of the $y$ value from the measured $y^{+}$are based on a standard (classical) scheme: HII regions are assumed to have a spherical structure in which the $\mathrm{He}^{+}$region, also spherical but smaller, is contained. Outside this $\mathrm{He}^{+}$ sphere, the $\mathrm{He}$ is neutral while the $\mathrm{H}$ is ionized, so that it is always $y^{+} \leq y$ (see e.g. Churchwell et al. 1974). Following this scheme, going outwards from the HII region, $y^{+}$should decrease or remain constant whether the ionizing star effective temperatures $\left(T_{\mathrm{e}}\right)$ are lower than $\sim 4 \times 10^{4} \mathrm{~K}$ or not. The measured $y^{+}$value depends on the angular resolution and on the frequency of observations (Churchwell et al. 1974). However, two unusual

* Appendices (Figs. 15, 16 and Sect. 4.1 "Carbon RRLs") are only available in electronic form at http://www . aanda.org features have been discovered (Roelfsema et al. 1987; Roelfsema et al. 1992; Adler et al. 1996) that fell outside this classical scheme. First, $y^{+}$showed a minimum toward the direction of the continuum radiation maximum (center) and then it increases toward the boundary of the HII region. For example, in component $\mathrm{n} .3$ of the source Sgr B2, $y^{+}$varied from $2.6 \%$ in the center to about $9 \%$ at the boundary (Roelfsema et al. 1987) Second, in the source W3A such increase yielded the unusually high value of $y^{+} \sim 20-40 \%$ (Roelfsema et al. 1992; Adler et al. 1996). The peculiar behaviour observed has been explained by Gulayev et al. (1997) as the result of an ionization structure which accounts for the ionization field hardening, influence of dust and He ionization from the metastable level $\left(2 S^{3}\right)$. It was also shown that in the presence of hot ionizing stars the $\mathrm{He}^{+}$ region can oversize the $\mathrm{H}^{+}$one, forming the so-called "inverse zone" $\left(\mathrm{He}^{+}-\mathrm{H}^{+}\right)$. These zones are thin $(<1 \%$ of the HII region size), however they can become thicker if a density gradient is present (Ershov et al. 1998).

The aim of this paper is to investigate the problem of how to determine $y$ from $y^{+}$on the basis of a well studied HII region, using new data and modeling. The Orion HII region has the required characteristics for such a task: it has been observed in a wide range of wavelengths; its ionizing stars are well known; its strong radio emission allows accurate RRL parameter determination. Furthermore, there are many model calculations (e.g. Shaver 1980; Rubin 1984) which predict the presence of density gradients in the Orion Nebula, that can be used as a basis for the new modeling. Nevertheless, various spectral observations of the Orion Nebula left the $y^{+}$problem open. H and He RRL mapping at $\sim 5 \mathrm{GHz}(109 \alpha$ transition, Jaffe \& Pankonin 1978; Pankonin et al. 1980) showed a $y^{+}$decreasing from the HII center outwards, according to the classical scheme. However, 
RRL observation at $22 \mathrm{GHz}(66 \alpha$, Tsivilev et al. 1986) showed an inverse dependence: $y^{+}$increases from $\sim 9 \%$ in the center to $\sim 11-12 \%$ at $\sim 3^{\prime}$ from the center. Tsivilev et al. (1986) explained the inverse behaviour on the bases of a "blister type" structure (i.e. not classical) with different $y^{+}$behaviour for the core and for the halo. The $y^{+}$decreasing with distance from the center at the lower frequency $(5 \mathrm{GHz})$ was explained by strong (but different for $\mathrm{H}$ and He RRLs) non-LTE effects, which are negligible at the higher $(22 \mathrm{GHz})$ frequency. According to their model, at greater distance from the center $\left(>3^{\prime}\right) y^{+}$decreased for both high and low frequencies. Gordon (1989) observed $\mathrm{H}$, $\mathrm{He} 40 \alpha \mathrm{RRL}(99.0 \mathrm{GHz})$ in the center of the nebula and in four positions shifted by $1^{\prime}$ from it, but individual $y^{+}$values were not presented. Nevertheless, using their spectra, $y^{+}=10.5 \%$ was evaluated for the center and the average $y^{+}=11 \%$ for the shifted positions was obtained, in agreement with the $22 \mathrm{GHz}$ observations. On the contrary, Peimbert et al. (1988) (41 $\alpha$ and 53 $\alpha$ RRL observations at 92 and $43 \mathrm{GHz}$ ) found $y^{+}$decreasing from the center outwards. In this case the telescope angular resolutions (18.5" and 39.5", respectively) were much better than for the above observations (2.6' and 1', Tsivilev et al. 1986; and Gordon et al. 1989, respectively) and consequently different size scales were characterised.

Similarly, at optical wavelengths, Peimbert \& TorresPeimbert (1977) found $y^{+}$decreasing from the center while Baldwin et al. (1991) obtained $y^{+}=8.8 \%$ nearly constant up to $\sim 5^{\prime}$ from the center.

Such a disagreement between and within optical and radio measurements of $y^{+}$has stimulated a joint project (between an Italian and a Russian group) to further investigate the ionization structure in the Orion Nebula in order to improve our knowledge of its physical parameters. The project was aimed at providing maps of hydrogen, helium and carbon RRL of this HII region at two frequencies with the same telescope beamwidth. This paper reports results obtained from the analysis of data from the first set of observations.

\section{Observations and data reduction}

The $66 \alpha$ (22 GHz frequency, Medicina $32 \mathrm{~m}$ VLBI dish) was pointed towards 14 positions and the $56 \alpha$ (36 GHz frequency, Pushchino RT-22) towards 10 positions in the Orion Nebula (see Fig. 1) and $\mathrm{H}, \mathrm{He}$ and $\mathrm{C}$ RRL were observed.

The angular resolution $\left(2^{\prime}\right)$ was the same for both telescopes, beam efficiencies $\left(\eta_{\mathrm{B}}\right)$ and system temperatures $\left(T_{\text {sys }}\right)$ were $\eta_{\mathrm{B}}=44 \%, T_{\text {sys }} \simeq 150 \mathrm{~K}$ and $\eta_{\mathrm{B}}=33 \%, T_{\text {sys }} \simeq 250 \mathrm{~K}$ for the Medicina and Pushchino radiotelescopes, respectively. All spectra were fitted with a Gaussian profile, using the nonlinear least square method (Smirnov \& Tsivilev 1982), and are presented in the Appendix.

\subsection{Medicina observations}

Observations were carried out with the autocorrelator of 16-25 MHz bandwidth (Comoretto \& Palagi 1993), and a velocity resolution of $0.654 \mathrm{~km} \mathrm{~s}^{-1}$, using the standard position switching technique with $4 \mathrm{~min}$ integration on source followed by 4 min integration on a reference position. The reference position was set at $1^{\circ}$ from the on source position to allow the beam retrace the same path of atmosphere of the on source position. Data were calibrated by observing DR21 (18.8 Jy, Dent 1972) every $40 \mathrm{~min}$. Spectra belonging to the same positions were calibrated and averaged. Integration times, for each position, span

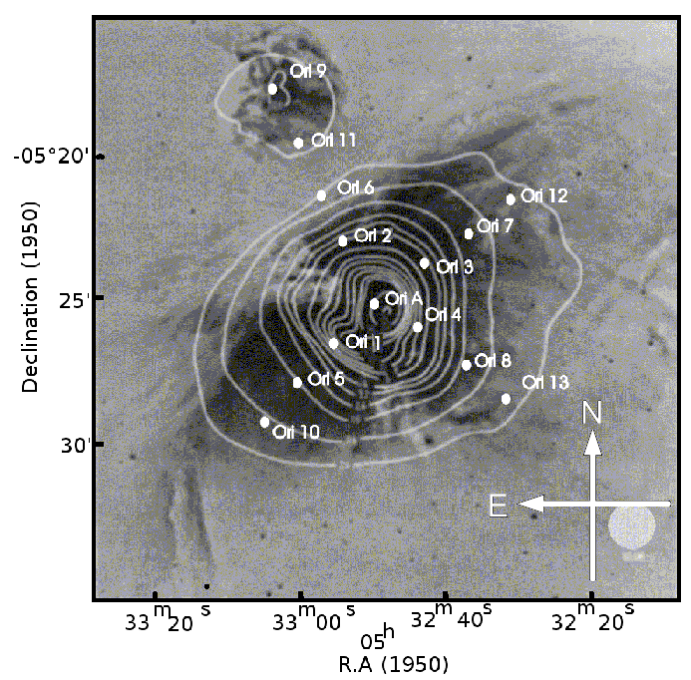

Fig. 1. Observed positions superimposed to on optical map ( $\mathrm{H} \alpha$ and [NII] - image, Hua \& Louise 1982) and on a $23 \mathrm{GHz}$ contour map (the circular spot represents the beam-size) of the Orion Nebula (Wilson \& Pauls 1984).

from 30 min to several hours depending on line strength: positions near the center (Ori A and positions from 1 to 4 ) required integration times of half an hour each. Otherwise external positions required integration times of up to $20 \mathrm{~h}$ each one. The whole project required more than $500 \mathrm{~h}$ of telescope time, carried out in several sessions from 1996 until 2001.

Table 1 contains the obtained line parameters, where $T_{\mathrm{L}}$ stands for brightness temperature.

\subsection{RT-22 observations}

Spectral and continuum simultaneous observations were carried out with a 128 channel, $64 \mathrm{MHz}$ bandwidth filter bank spectral analyser $\left(4.11 \mathrm{~km} \mathrm{~s}^{-1}\right.$ velocity resolution). A ON-ON (Berulis et al. 1983) observing technique based on beam switching was adopted, with $\sim 5 \mathrm{~min}$ on source integration time. To minimise the spectra quasi-sinusoidal base line shape due to standing-waves produced by reflections in the antenna system, the observations were made: a) by $\pm \lambda / 8$ focus shifting every 15-20 min and b) by several sessions per year in different seasons, from 1995 to 2001. The spectra obtained during one day observations at each position have been averaged. These intermediate spectra were averaged after the subtraction of the continuum level; in this way for each position the final spectra were obtained for the whole observation period. Integration times spanned from 7 to $10 \mathrm{~h}$ for Ori A and positions 1-4, while for positions 5-9 they spanned from 21 to $25 \mathrm{~h}$.

Table 2 presents the line parameter and continuum temperature, where $T_{\mathrm{C}}$ and $T_{\mathrm{L}}$ represent brightness temperatures.

\section{Results and discussion}

We call rings the set of observed positions having the same angular distance from the center: Ring 1 includes positions 1-4, Ring 2 positions 5-8 and Ring 3 position 10-13, placed respectively at $2^{\prime}, 4^{\prime}$ and $6^{\prime}$ from the center (i.e. star $\theta^{1}$ Ori $C$ ). All the spectra belonging to Ring 3 are not considered in the He lines analysis because of poor signal-to-noise ratio. The $y^{+}$weighted average of $56 \alpha$ and $66 \alpha$ data are presented. 
Table 1. Results from $66 \alpha$ line observations.

\begin{tabular}{|c|c|c|c|c|c|c|c|}
\hline Pos. & $\mathrm{Ln}$ & $\begin{array}{c}T_{\mathrm{L}} \\
(\mathrm{mK})\end{array}$ & $\begin{array}{l}F W H M \\
\left(\mathrm{~km} \mathrm{~s}^{-1}\right)\end{array}$ & $\begin{array}{c}V_{\mathrm{LSR}} \\
\left(\mathrm{km} \mathrm{s}^{-1}\right)\end{array}$ & $\begin{array}{c}y^{+} \\
(\%)\end{array}$ & $\begin{array}{c}\mathrm{AR}(\mathrm{h}, \mathrm{m}, \mathrm{s}) \\
(1950)\end{array}$ & $\begin{array}{c}\operatorname{Dec}\left({ }^{\circ},{ }^{\prime \prime},{ }^{\prime \prime}\right) \\
(1950)\end{array}$ \\
\hline \multirow[t]{3}{*}{$\overline{\text { Ori A }}$} & $\mathrm{H}$ & $4163(12)$ & $25.66(0.09)$ & $-1.74(0.4)$ & & 53249 & -52516 \\
\hline & $\mathrm{He}$ & $440(14)$ & $18.82(0.74)$ & $-3.6(0.4)$ & $7.7(0.6)$ & & \\
\hline & $\mathrm{C}$ & $167(27)$ & (4.4) & $+10.3(0.5)$ & & & \\
\hline \multirow[t]{3}{*}{ Ori 1} & $\mathrm{H}$ & $1898(15)$ & $28.06(0.23)$ & $-1.06(1.0)$ & & 53255.0 & -52628 \\
\hline & $\mathrm{He}$ & 201(16) & $23.22(2.91)$ & $+0.1(1.1)$ & $8.8(1.4)$ & & \\
\hline & $\mathrm{C}$ & $167(28)$ & $4.48(1.17)$ & $+11.45(0.5)$ & & & \\
\hline \multirow[t]{3}{*}{ Ori 2} & $\mathrm{H}$ & $1864(15)$ & $27.30(0.24)$ & $-3.25(0.40)$ & & 53253.5 & -52307 \\
\hline & $\mathrm{He}$ & $222(17)$ & $21.5(2.4)$ & $-2.39(0.9)$ & $9.5(1.3)$ & & \\
\hline & $\mathrm{C}$ & $60(26)$ & $6.9(3.7)$ & $+7.8(1.5)$ & & & \\
\hline \multirow[t]{3}{*}{ Ori 3} & $\mathrm{H}$ & $2725(26)$ & $25.60(0.25)$ & $-4.43(0.40)$ & & 53243.5 & -52358 \\
\hline & $\mathrm{He}$ & $402(34)$ & $15.8(1.8)$ & $-5.32(0.8)$ & $9.1(1.4)$ & & \\
\hline & $\mathrm{C}$ & $237(48)$ & $5.7(1.5)$ & $+8.65(0.70)$ & & & \\
\hline \multirow[t]{3}{*}{ Ori 4} & $\mathrm{H}$ & 1988(19) & $27.89(0.28)$ & $-3.66(0.41)$ & & 53244.0 & -52605 \\
\hline & $\mathrm{He}$ & $224(21)$ & $21.65(2.55)$ & $-6.26(1.0)$ & $8.7(1.3)$ & & \\
\hline & $\mathrm{C}$ & $75(38)$ & $3.95(2.5)$ & $+3.78(1.1)$ & & & \\
\hline \multirow[t]{3}{*}{ Ori 5} & $\mathrm{H}$ & $447.4(7.4)$ & $29.12(0.44)$ & $-2.50(0.44)$ & & 53300.0 & -52800 \\
\hline & $\mathrm{He}$ & 59.5.(7.9) & $18.0(3.8)$ & $-4.2(1.4)$ & $8.2(2.1)$ & & \\
\hline & $\mathrm{C}$ & $31.5(12.0)$ & $6.0 \mathrm{fix}(3.0)$ & $+9.3(1.2)$ & & & \\
\hline \multirow[t]{3}{*}{ Ori 6} & $\mathrm{H}$ & $456.1(6.7)$ & $26.63(0.33)$ & $-0.58(0.43)$ & & 53256.7 & -52130 \\
\hline & $\mathrm{He}$ & $64.1(6.5)$ & $17.0(2.0)$ & $+1.40(0.80)$ & $9.0(1.4)$ & & \\
\hline & $\mathrm{C}$ & $74.1(9.3)$ & $4.76(0.73)$ & $+8.3(0.5)$ & & & \\
\hline \multirow[t]{3}{*}{ Ori 7} & $\mathrm{H}$ & $531.1(9.8)$ & $28.0(0.40)$ & $-6.6(0.5)$ & & 53236.5 & -52258 \\
\hline & $\mathrm{He}$ & $51.7(5.8)$ & $19.3(4.1)$ & $-6.0(1.6)$ & $6.4(1.7)$ & & \\
\hline & $\mathrm{C}$ & $40.2(17.2)$ & $2.75(1.4)$ & $+8.8(0.7)$ & & & \\
\hline \multirow[t]{2}{*}{ Ori 8} & $\mathrm{H}$ & $395.2(4.9)$ & $28.96(0.43)$ & $-4.69(0.44)$ & & 53237.5 & -52720 \\
\hline & $\mathrm{He}$ & $34.3(6.3)$ & $17.6(3.9)$ & $-4.80(1.65)$ & $5.3(1.5)$ & & \\
\hline \multirow[t]{3}{*}{ Ori 9} & $\mathrm{H}$ & $240.7(7.2)$ & $23.2(0.7)$ & $+8.4(0.6)$ & & 53304.0 & -51755 \\
\hline & $\mathrm{He}$ & $24.7(6.9)$ & $17.6(8.3)$ & $+6.2(2.4)^{*}$ & $7.8(3.2)$ & & \\
\hline & $\mathrm{C}$ & $43.6(12.8)$ & $4.1(1.4)^{*}$ & $+11.0(0.8)$ & & & \\
\hline \multirow[t]{3}{*}{ Ori10 } & $\mathrm{H}$ & $263.3(7.0)$ & $27.8(0.75)$ & $-2.37(0.50)$ & & 53305.0 & -52918 \\
\hline & $\mathrm{He}$ & $13.1(8.2)$ & $19.7(15.3)$ & $-5.2(4.8)$ & $3.5(3.5)$ & & \\
\hline & $\mathrm{C}$ & $52.9(20.5)$ & $1.5(0.7)$ & $+(9.5)$ & & & \\
\hline \multirow[t]{3}{*}{ Ori11 } & $\mathrm{H}$ & $211.5(3.8)$ & $25.1(0.6)$ & $+3.9(0.5)$ & & 53300.0 & -51937 \\
\hline & $\mathrm{He}$ & $6.2(4.1)$ & $20.7(16.0)$ & $+3.5^{*}$ & $\sim 2.4$ & & \\
\hline & $\mathrm{C}$ & $47.0(7.7)$ & $4.4(0.9)$ & $+8.1(0.60)$ & & & \\
\hline \multirow[t]{3}{*}{ Ori12 } & $\mathrm{H}$ & $213.7(5.8)$ & $28.9(1.0)$ & $-5.9(0.60)$ & & 53231.0 & -52152 \\
\hline & $\mathrm{He}$ & $36.3(6.3)$ & $18.8(4.5)$ & $-3.6(1.6)$ & $11.0(3.3)^{*}$ & & \\
\hline & $\mathrm{C}$ & $26.0(13.2)$ & $3.6(2.2)^{*}$ & $+8.3(1.0)$ & & & \\
\hline \multirow[t]{3}{*}{ Ori13 } & $\mathrm{H}$ & $145.1(6.5)$ & $35.7(1.4)$ & $-3.2(0.70)$ & & 53232.0 & -52830 \\
\hline & $\mathrm{He}$ & $15.3(4.8)$ & $13.1(8.8)$ & $-9.5(3.1)$ & $3.9(3.0)$ & & \\
\hline & $\mathrm{C}$ & $17.5(11.0)$ & $4.1(3.1)^{*}$ & $+8.1(1.2)$ & & & \\
\hline
\end{tabular}

* Fixed parameter under the fitting.

\subsection{HII region structure and kinematics}

We consider the velocity $\left(V_{\mathrm{LSR}}\right)$ and line width $(\Delta V)$ values of hydrogen lines as a function of the distance from the center, from the South-East toward the North-West (Figs. 3 and 4) and from North-East toward the South-West (Figs. 5 and 6).

In Fig. 7 we plot the $V_{\mathrm{LSR}}$ as a function of the distance from the center, averaging values at the same distance. On the other hand, the hydrogen line width (Fig. 8) increases going out of the nebula. This picture emphasizes the systematic motion of the ionized gas.

Such a picture is the opposite of the one we would expect for a spherical symmetry structure of the HII region, where the maximum of $\Delta V$ and the most negative $V_{\mathrm{LSR}}$ should be observed in the center. This situation can be naturally explained assuming a "blister-type" model (Zuckerman 1973; Balick et al. 1974; Pankonin et al. 1979; Tsivilev et al. 1986; Rubin 1991), where the HII region formed near the boundary of a molecular cloud facing the observer and has an asymmetric expansion structure, expanding mainly toward the observer, either along the line of sight or with a small inclination angle (Pankonin et al. 1979). Towards this direction, into the molecular cloud (see Fig. 9), the dense core is formed with a much smaller size than that of the low-density halo, which is expanding in the opposite direction. Thus, towards the central part the main contribution to the lines profile is given by the dense core, which has some positive $V_{\mathrm{LSR}}$ due to its motion towards the molecular cloud, away from the observer. In this way the core positive $V_{\mathrm{LSR}}$ compensates the negative $V_{\text {LSR }}$ of the halo: less negative velocities will be observed toward the center than in the outer positions. This effect will be amplified by the existing central "bubble" around the exciting stars (Pankonin et al. 1979). Also, both these effects will lead to line widths increasing with distance from the center, that can be accompanied by a possible acceleration of the expansion with radius favoured by the expansion of the ionized gas from the molecular cloud into outer space (Bodenheimer et al. 1979). This also explains the line width increasing far from the center (up to $\sim 6^{\prime}$ ). 
Table 2. Results from $56 \alpha$ line and continuum observations.

\begin{tabular}{|c|c|c|c|c|c|c|c|}
\hline Pos. & $\begin{array}{c}T_{\mathrm{C}} \\
(\mathrm{mK}) \\
\end{array}$ & Line & $\begin{array}{c}T_{\mathrm{L}} \\
(\mathrm{mK})\end{array}$ & $\begin{array}{l}F W H M \\
\left(\mathrm{~km} \mathrm{~s}^{-1}\right)\end{array}$ & $\begin{array}{c}V_{\mathrm{lsr}} \\
\left(\mathrm{km} \mathrm{s}^{-1}\right) \\
\end{array}$ & $\begin{array}{c}y^{+} \\
(\%) \\
\end{array}$ & $\begin{array}{c}T_{\mathrm{e}}(\mathrm{LTE}) \\
K \\
\end{array}$ \\
\hline \multirow[t]{3}{*}{ Ori A } & $4758(3)$ & $\overline{\mathrm{H}}$ & 1900(16) & $25.51(0.24)$ & $-1.6(0.3)$ & & $8790(220)$ \\
\hline & & $\mathrm{He}$ & 228.2(18.5) & $19.3(2.1)$ & $-1.6(0.8)$ & $8.7^{a}(0.7)$ & \\
\hline & & $\mathrm{C}$ & $83(66)$ & $2.9^{*}$ & $+10.3(1.4)$ & & \\
\hline \multirow[t]{3}{*}{ Ori 1} & $2548(3)$ & $\mathrm{H}$ & $936.1(13.1)$ & $27.2(0.49)$ & $-1.7(0.4)$ & & $8900(280)$ \\
\hline & & $\mathrm{He}$ & $100.2(14.1)$ & $23.3(4.4)$ & $-5.9(2.0))$ & $9.1(2.2)$ & \\
\hline & & $\mathrm{C}$ & $101.8(55.6)$ & $3.4^{*}$ & $+11.7(1.0)(0.4)$ & & \\
\hline \multirow[t]{3}{*}{ Ori 2} & 2181(3) & $\mathrm{H}$ & $799.4(10.5)$ & $26.59(0.42)$ & $-1.8(0.4)$ & & $9000(250)$ \\
\hline & & $\mathrm{He}$ & $92.8(10.7)$ & $24.3(4.1)$ & $-0.9(1.7)$ & $10.6(1.8)$ & \\
\hline & & $\mathrm{C}$ & 30 & $2.85^{*}$ & $+10.5^{*}$ & & \\
\hline \multirow[t]{3}{*}{ Ori 3} & $2116(3)$ & $\mathrm{H}$ & $902.8(12.0)$ & $25.58(0.35)$ & $-3.4(0.3)$ & & $8300(220)$ \\
\hline & & $\mathrm{He}$ & 130.9(11.6) & $21.2(2.8)$ & $-3.9(1.2)$ & $12.0(1.9)$ & \\
\hline & & $\mathrm{C}$ & $62(49)$ & $3.3^{*}$ & $+10.0(1.3)$ & & \\
\hline \multirow[t]{3}{*}{ Ori 4} & 2671(3) & $\mathrm{H}$ & $1138.8(10.8)$ & $27.37(0.30)$ & $-3.0(0.4)$ & & $8000(160)$ \\
\hline & & $\mathrm{He}$ & $160.0(13.2)$ & $17.2(1.9)$ & $-1.7(0.8)$ & $8.8(1.2)$ & \\
\hline & & $\mathrm{C}$ & $157(46)$ & $2.85^{*}$ & $+10.4(0.5)$ & & \\
\hline \multirow[t]{3}{*}{ Ori 5} & $820.3(3)$ & $\mathrm{H}$ & $280.0(5.2)$ & $27.8(0.65)$ & $-2.7(0.4)$ & & $9570(400)$ \\
\hline & & $\mathrm{He}$ & $20.3(6.2)$ & $18.6(9.2)$ & $+0.16(3.6)$ & $4.8(2.8)$ & \\
\hline & & $\mathrm{C}$ & $46.2(40.0)$ & $3.0^{*}$ & $+9.1(2.2)$ & & \\
\hline \multirow[t]{3}{*}{ Ori 6} & $664.0(2)$ & $\mathrm{H}$ & $270.7(6.5)$ & $27.74(0.80)$ & $-0.6(0.6)$ & & $8240(530)$ \\
\hline & & $\mathrm{He}$ & $19.4(6.5)$ & $28.8(12.3)$ & $-3.0^{*}$ & $7.8(5.2)$ & \\
\hline & & $\mathrm{C}$ & $51(15)$ & $5.7 *$ & $+10.1(1.7)$ & & \\
\hline \multirow[t]{4}{*}{ Ori 7} & $455.1(1.0)$ & $\mathrm{H}$ & $172.3(4.3)$ & $28.65(0.88)$ & $-5.1(0.6)$ & & $8300(440)$ \\
\hline & & $\mathrm{He}$ & $35.1(6.2)$ & $15.7(4.7)$ & $-6.2(2.0)$ & $11.2(3.4)$ & \\
\hline & & $\mathrm{C} 1$ & 98 & $4.9^{*}$ & +9.9 & & \\
\hline & & $\mathrm{C} 2$ & 77 & $1.0^{*}$ & -1.7 & & \\
\hline \multirow[t]{3}{*}{ Ori 8} & $686.2(1.0)$ & $\mathrm{H}$ & $280.0(5.8)$ & $30.8(0.7)$ & $-5.0(0.5)$ & & $7770(350)$ \\
\hline & & $\mathrm{He}$ & $25.8(7.1)$ & $21.7(9.0)$ & $-3.3(3.9)$ & $6.5(3.2)$ & \\
\hline & & $\mathrm{C}$ & $40(22)$ & $4.5^{*}$ & +9.1(1.4) & & \\
\hline \multirow[t]{3}{*}{ Ori 9} & $240.5(1.0)$ & $\mathrm{H}$ & $150.2(5.5)$ & $20.1(0.8)$ & $+7.2(0.6)$ & & $7890(610)$ \\
\hline & & $\mathrm{He}$ & $10.5(5.5)$ & 18.3(11.6) & $+6.9^{*}$ & $6.3(5.3)$ & \\
\hline & & $\mathrm{C}$ & $52(19)$ & $2.85^{*}$ & $+7.3(1.0)$ & & \\
\hline
\end{tabular}

${ }^{a}$ The average value from this $500 \mathrm{kHz}$ frequency resolution observation and from the $125 \mathrm{kHz}$ frequency one; both were with RT-22. * Fixed parameter under the fitting.

Moreover, in this scheme (Fig. 9) the core should be ionization-bounded while the halo could be either ionizationbounded or density-bounded. The latter case yields $y_{\text {core }}^{+}<y_{\text {halo }}^{+}$, that can explain our $y^{+}(R)$ data. In summary, the combination of the obtained $\left(V_{\mathrm{LSR}}(R), \Delta V(R)\right.$ and $\left.y^{+}(R)\right)$ data qualitatively supports the "blister-type" model.

\subsection{Ionized helium distribution}

The distribution of $y^{+}$is shown in Fig. 10 to Fig. 13 .

A $y^{+}$maximum can be seen towards the north of the center (star Ori $\theta^{1} \mathrm{C}$ ), decreasing towards the outer part of the nebula, more evident in the southern direction. This picture is in agreement with the results of Pankonin et al. (1980), who found similar different $y^{+}$behaviours along different directions with a strong decrease towards the southeast.

It is also in agreement with the optical data of Peimbert \& Torres-Peimbert (1977) and Baldwin et al. (1991) who observed in different directions, obtaining different $y^{+}$behaviours. Furthermore, the $y^{+}$maximum does not coincide with the center. The position is in agreement with the $y^{+}$increasing trend towards north-east as derived by Lockman \& Brown (1982) (76 $\alpha$ lines), who found an $y^{+}$increase from $\approx 8 \%$ in the center up to $\approx 11 \%$ at a distance of $\sim 2.5^{\prime}$.

Figure 14 shows the $y^{+}(R)$ behaviours (averaged over each ring, Table 3 ), as a function of angular distance (R) from the center. There is atentative evidence of variations in $y^{+}:$a minimum in the center, then $y^{+}$increases with distance up to $2^{\prime}$ from the center, then it decreases again. From Figs. 10-13 one can see that such $y^{+}(R)$ behaviour is clearer for all directions.

This scenario seems to confirm the asymmetric model (Tsivilev et al. 1986) where the $\mathrm{He}^{+}$zone size is smaller than the $\mathrm{H}^{+}$, but the ratio of their sizes is smaller in the core than in the envelope, yielding $\left(y_{\text {core }}^{+}<y_{\text {halo }}^{+}\right)$.

On the other hand, Peimbert et al. (1988) found $y^{+}$decreasing from the center as $y^{+} \approx 9 \%$ at $\sim 0.5^{\prime}$ and $y^{+} \approx 7 \%$ at $\sim 1^{\prime}$ (the angular resolutions were $18.5^{\prime \prime}$ and $39.5^{\prime \prime}$, respectively). The calculated average value over all positions, which lies approximately in the frame of our one beam in the Ori A direction, gives $y^{+}=8.2( \pm 0.3) \%$, in agreement with our Ori A value. In principle, the decrease that they found can be due to an intermediate minimum which might be seen with higher angular resolution in a transition zone between the core and the halo (envelope) because $i$ ) the $y^{+}(R)$ should decrease more steeply for the core than for the halo and $i$ ) if a core-halo boundary is sharp, an observer will obtain an average of both $y_{\text {core }}^{+}$and $y_{\text {halo }}^{+}$values (weighted by their emission measures). This can explain the $y^{+}$minimum: the smaller the beam size, the deeper will be the minimum measured. Such scenario is compatible with a "blister-type" structure. Optical data (Baldwin et al. 1991) however did not show any $y^{+}$minimum at similar angular distances $\left(\sim 1^{\prime}\right)$, so the question of the core-halo boundary sharpness is still unsolved. To attempt to answer this question, high angular resolution observations are required. 

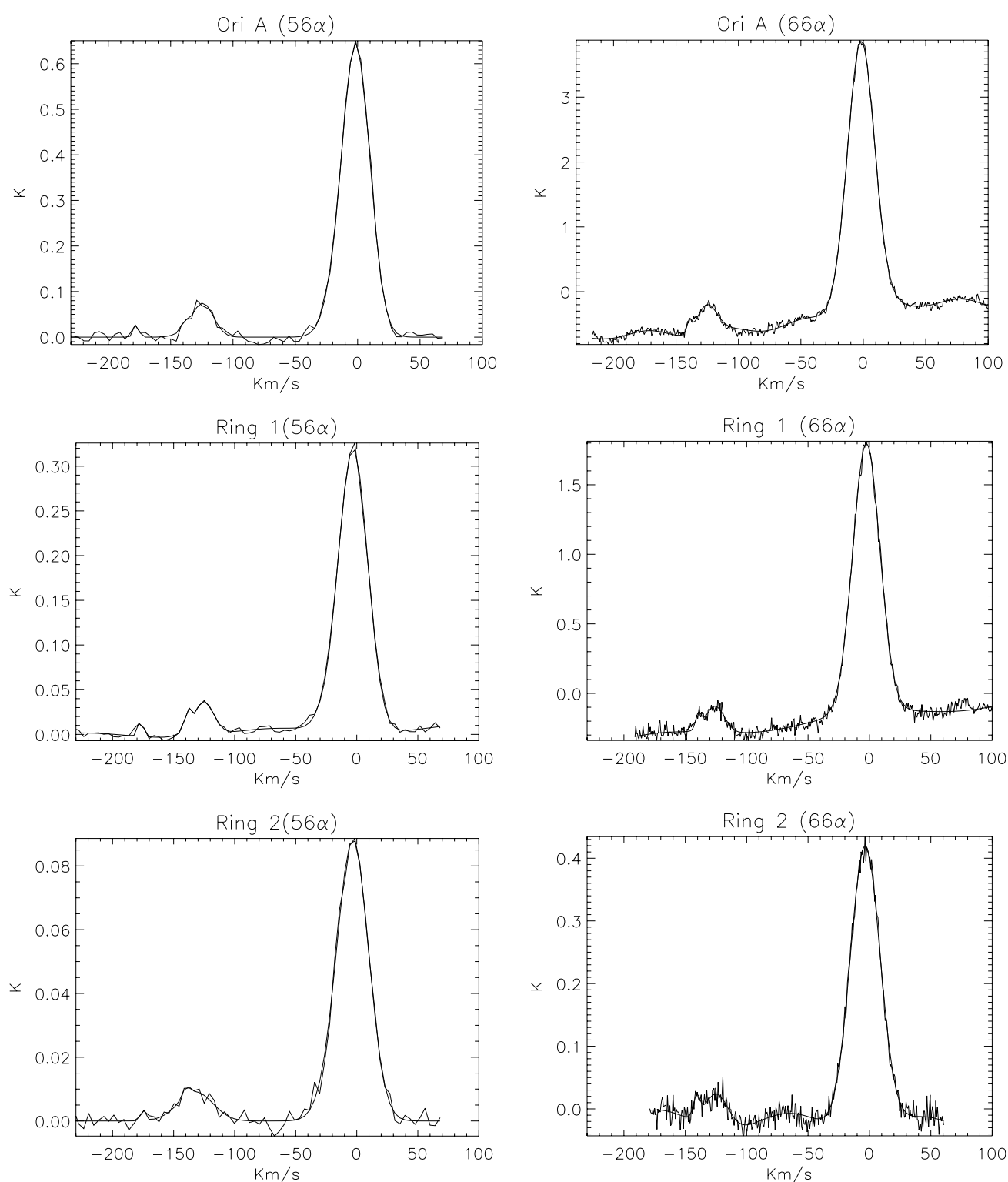

Fig. 2. A sample of the obtained spectra: left panels the $\mathrm{H}, \mathrm{He}, \mathrm{C} 56 \alpha(8 \mathrm{~mm}) \mathrm{RRL}$ lines, on the right the $\mathrm{H}, \mathrm{He}, \mathrm{C} 66 \alpha(13.5 \mathrm{~mm}) \mathrm{RRL}$ lines. Upper panel presents the central position data (Orion A), middle panel - the Ring 1 data and lower panel - the Ring 2 data (see text). On the $Y$-axis antenna temperatures $(\mathrm{K})$ are plotted, while on the $x$-axis are plotted velocity terms, where: $V_{\mathrm{LSR}} \approx 0 \mathrm{~km} \mathrm{~s}^{-1}-$ is the hydrogen line position $(\mathrm{H})$, $V_{\mathrm{LSR}} \approx-122 \mathrm{~km} \mathrm{~s}^{-1}$ is the helium line position $(\mathrm{He}), V_{\mathrm{LSR}} \approx-140 \mathrm{~km} \mathrm{~s}^{-1}$ is the carbon line position (C) and $V_{\mathrm{LSR}} \approx-180 \mathrm{~km} \mathrm{~s}^{-1}$ is the molecular line position, OCS, for the $8 \mathrm{~mm}$ observations.

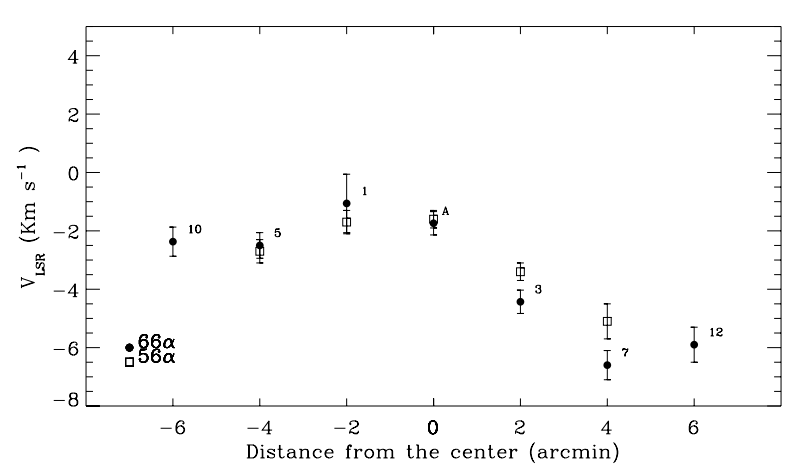

Fig. 3. $V_{\text {LSR }}$ from South-East to North West.

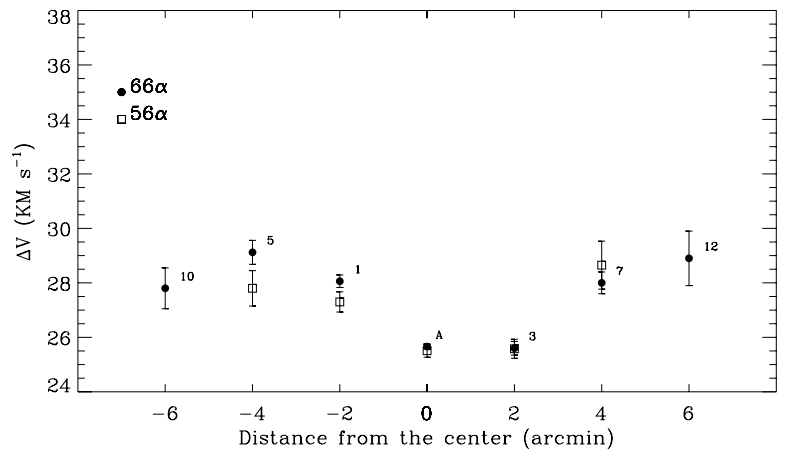

Fig. 4. $\Delta V$ from South-East to North West. 


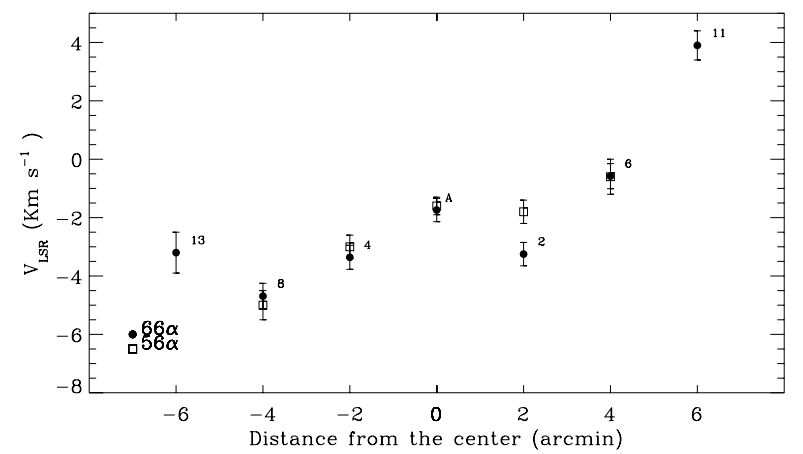

Fig. 5. $V_{\text {LSR }}$ from South-West to North-East.

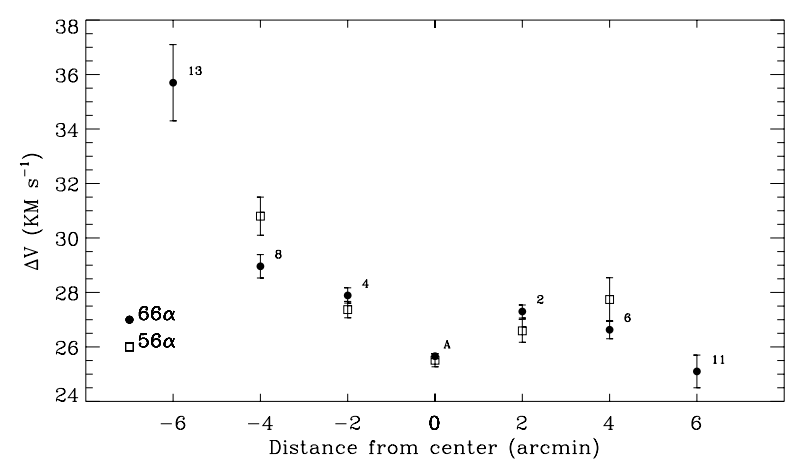

Fig. 6. $\Delta V$ from South-West to North-East.

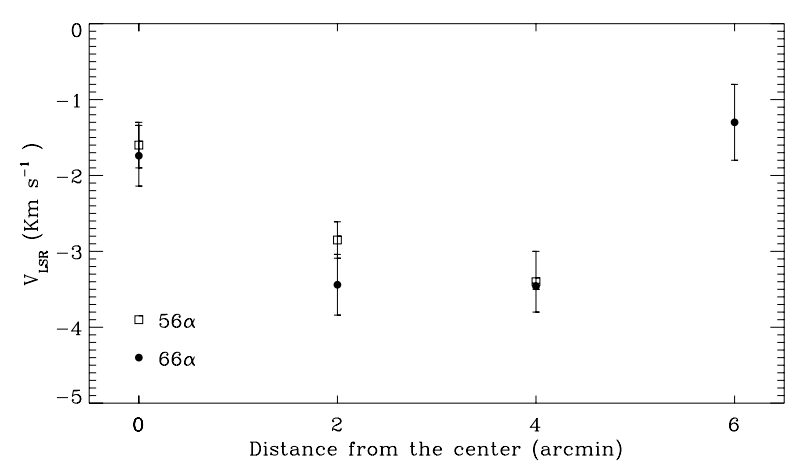

Fig. 7. $V_{\text {LSR }}$ from $\mathrm{H}$ lines.

However, many systematic effects could influence the measurement and the interpretation of optical data (Izotov \& Thuan 2004; Pagel 2000). On the other hand, such effects are reduced if both singlet helium lines, which are more hydrogenic than triplets lines, and hydrogen lines with transitions $n>2$ are considered. Indeed, the ratio of lines $\mathrm{He}(\lambda 6678) / \mathrm{H}_{11-3}$ (see Table 6 and Fig. 12 of Baldwin et al. 1991) gives an $y^{+}(R)$ behaviour similar to ours.

Nevertheless, our $y^{+}$averaged values (solid line in Fig. 14 and Table 3) are in agreement, within the errors, with the $y^{+}=$ $8.8( \pm 0.6) \%$ value reported by Baldwin et al. (1991).

\subsection{Helium abundance}

Since the $\mathrm{He}^{+}$region size is smaller than the $\mathrm{H}^{+}$one, it is necessary to correct the observed $y^{+}$values for the ionization structure to obtain a true helium abundance. However, according to a "blister-type" model (Tsivilev 1991; Rubin 1991) either part of the envelope or all of it can be density limited, that is most ionizing photons can escape freely. In this case the size of both $\mathrm{He}^{+}$

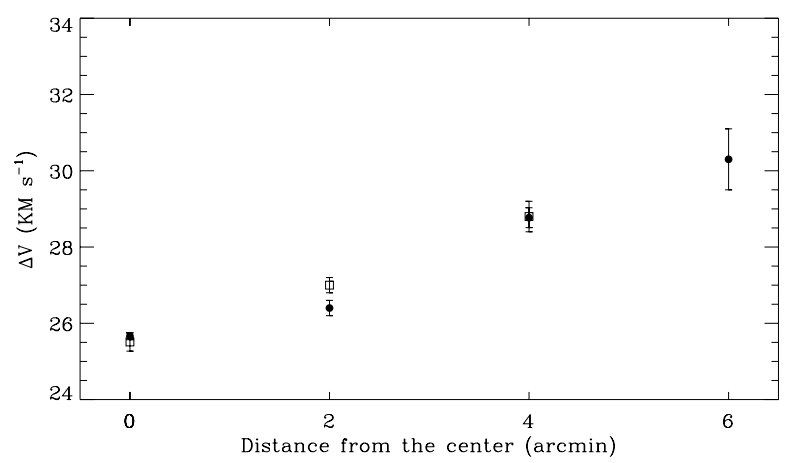

Fig. 8. Hydrogen line widths.

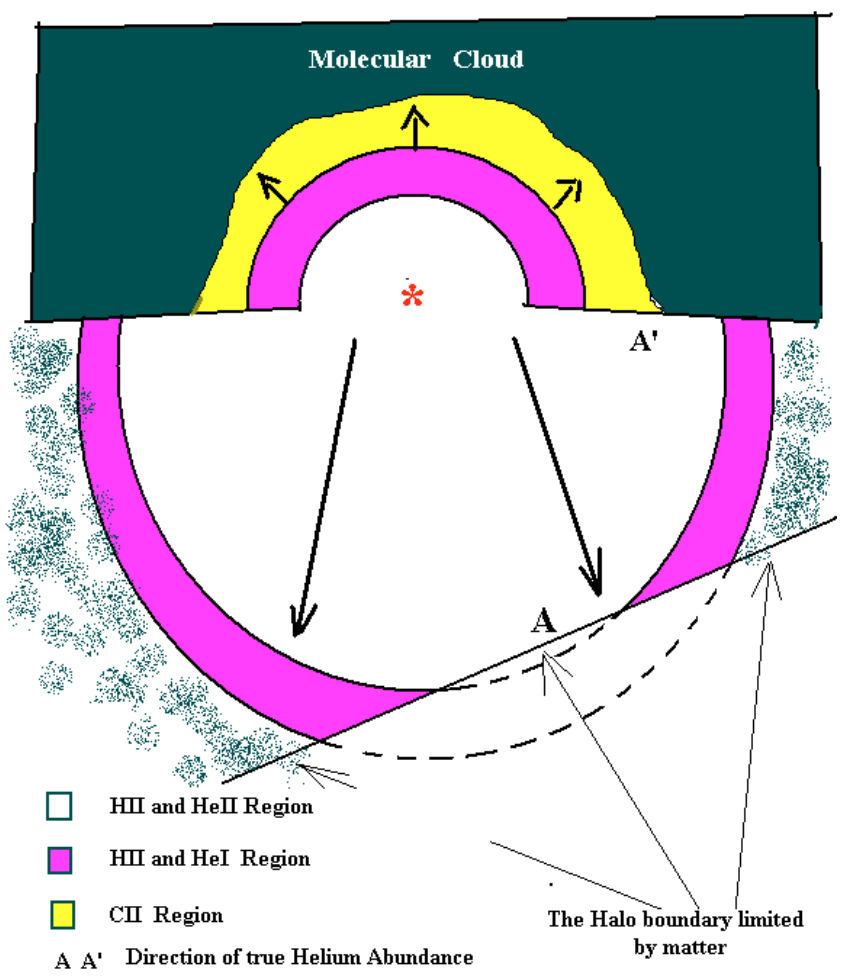

Fig. 9. The blister type model.

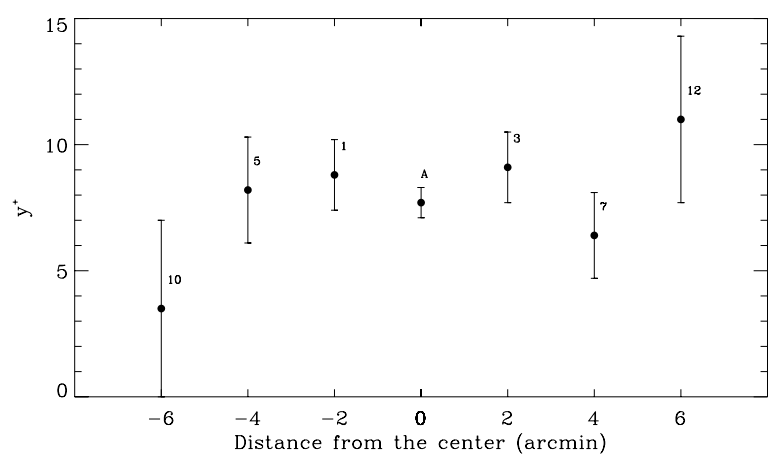

Fig. 10. $y^{+}$from $66 \alpha$ lines from South-East toward the North-West. Labels near plotted points indicate individual positions (Table 1).

and $\mathrm{H}^{+}$zones coincide and the measured helium abundance is true in that place (direction $\mathrm{A}-\mathrm{A}^{\prime}$ in Fig. 9). These conditions would be matched in the region where a maximum of $y^{+}$has been measured.

Under this hypothesis a "true" value of $y=10.0( \pm 0.8) \%$ has been obtained with a weighted average of $56 \alpha$ and $66 \alpha$ data 


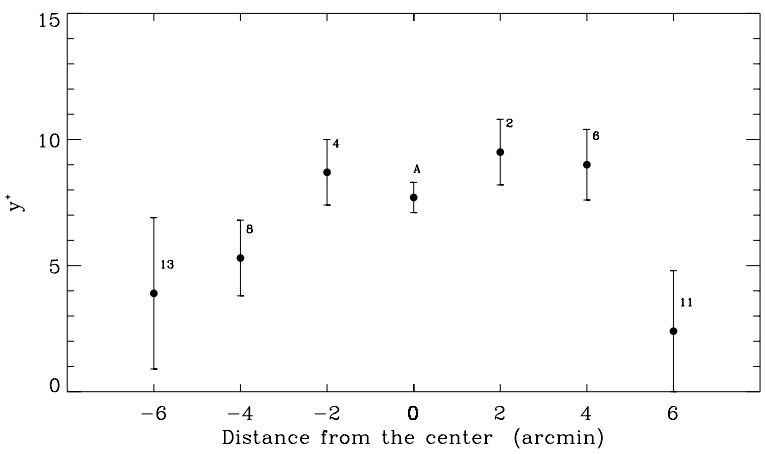

Fig. 11. $y^{+}$from $66 \alpha$ lines from South-West toward the North-East. Labels near plotted points indicate individual positions (Table 1).

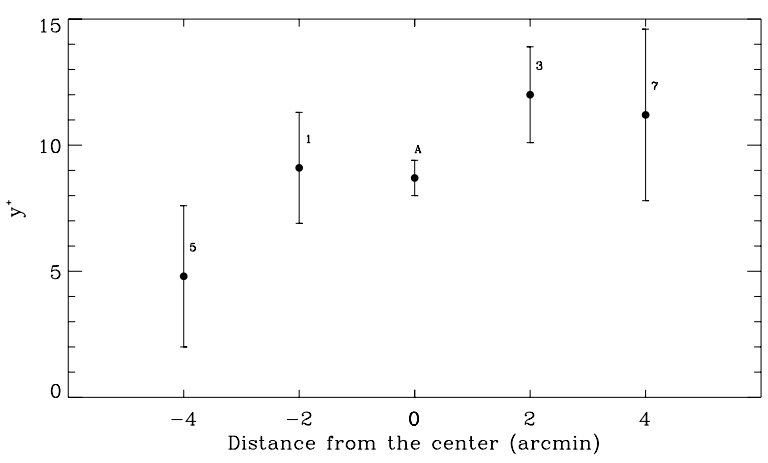

Fig. 12. $y^{+}$from $56 \alpha$ lines from South-East toward the North-West. Labels near plotted points indicate individual positions (Table 2).

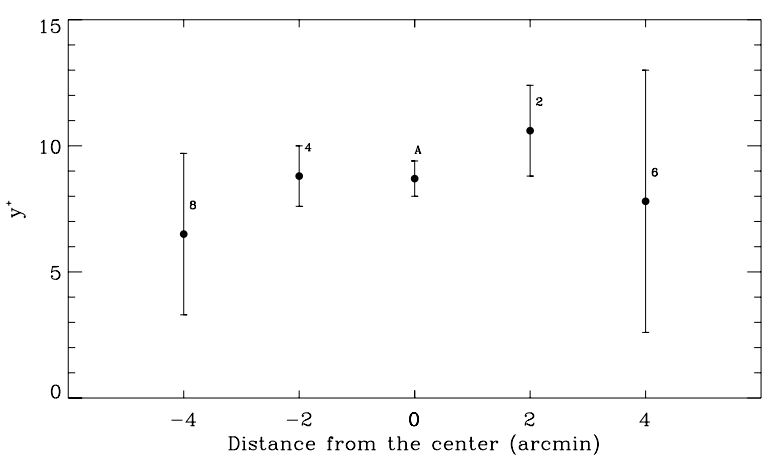

Fig. 13. $y^{+}$from $56 \alpha$ lines from South-West toward the North-East. Labels near plotted points indicate individual positions (Table 2).

of positions 2 and 3 , where the $y^{+}$has the maximum (see Figs. 10-13 ). This value is in good agreement with optical values: $y=10.0( \pm 0.5) \%$ (Peimbert \& Torres-Peimbert 1977) and $y=9.8( \pm 0.5) \%$ (Esteban et al. 1998), where the correction for ionization structure was made.

Further, one can evaluate the primordial helium abundance. The abundance of heavier elements $(Z=0.011 \pm 0.0022)$ for Orion A was measured by Baldwin et al. (1991). Using the last available value $\Delta Y / \Delta Z=2.8( \pm 0.5)$ for HII regions reported by Izotov \& Thuan (2004), the obtained helium abundance can be corrected for the stellar nucleosythesis contribution. The determined primordial helium abundance (by mass) is therefore $Y_{\mathrm{p}}=25.2( \pm 1.8) \%$.

\subsection{Electron temperature}

Measured LTE electron temperatures $\left(T_{\mathrm{e}}(\mathrm{LTE})\right)$ show an interesting tendency: West points $(3,4,7,8)$ have lower temperatures

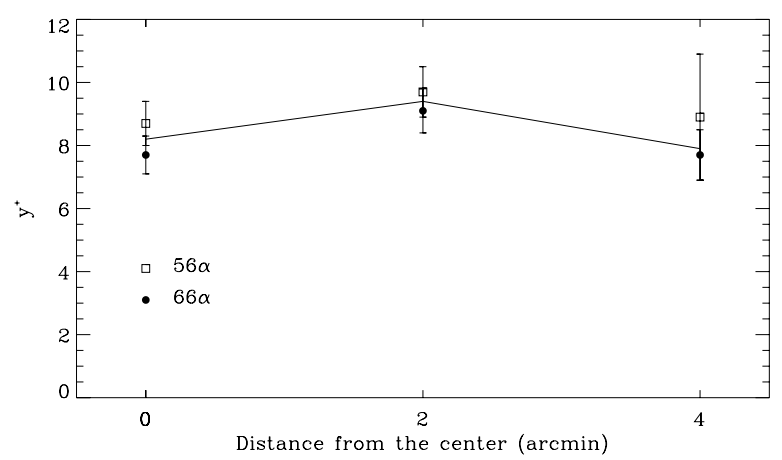

Fig. 14. $y^{+}(\%)$ averaged over the rings. The data for the $56 \alpha$ are presented separately from the $66 \alpha$ transition and for their average (solid line).

Table 3. $y^{+}$and hydrogen line parameters as a function of the distance from the center.

\begin{tabular}{|c|c|c|c|c|}
\hline & $0^{\prime}$ & $2^{\prime}$ & $4^{\prime}$ & $6^{\prime}$ \\
\hline \multicolumn{5}{|c|}{$y^{+}(\%)$} \\
\hline $56 \alpha$ & $8.7(0.7)$ & $9.7(0.8)$ & $8.9(2.0)$ & - \\
\hline $66 \alpha$ & $7.7(0.6)$ & $9.1(0.7)$ & $7.7(0.8)$ & - \\
\hline Average & $8.2(0.46)$ & $9.4(0.5)$ & $7.9(0.7)$ & - \\
\hline \multicolumn{5}{|c|}{$\mathrm{H}$ line $V_{\mathrm{LSR}}\left(\mathrm{km} \mathrm{s}^{-1}\right)$} \\
\hline $56 \alpha$ & $-1.6(0.3)$ & $-2.85(0.24)$ & $-3.4(0.4)$ & - \\
\hline $66 \alpha$ & $-1.74(0.4)$ & $-3.44(0.40)$ & $-3.46(0.4)$ & $-1.3(0.5)$ \\
\hline \multicolumn{5}{|c|}{$\mathrm{H}$ line $\Delta V\left(\mathrm{~km} \mathrm{~s}^{-1}\right)$} \\
\hline $56 \alpha$ & $25.51(0.24)$ & $27.0(0.2)$ & $28.8(0.4)$ & - \\
\hline $66 \alpha$ & $25.66(0.09)$ & $26.4(0.2)$ & $28.77(0.26)$ & $30.3(0.8)$ \\
\hline
\end{tabular}

than the East ones. On average, East points have $T_{\mathrm{e}}(\mathrm{LTE})=$ $9000 \mathrm{~K}$, while West ones have $T_{\mathrm{e}}(\mathrm{LTE})=8090 \mathrm{~K}$. The difference between these two values falls outside the experimental errors.

From $T_{\mathrm{e}}(\mathrm{LTE})$ it is possible to obtain the real electronic temperature $\left(T_{\mathrm{e}} \simeq b_{n}^{0.87} T_{\mathrm{e}}\right.$ (LTE) where $b_{n}$ values are from Salem $\&$ Brocklehurst 1979). If we assume the electron densities to be $N_{\mathrm{e}} \sim 10^{4} \mathrm{~cm}^{-3}$ at the center, $N_{\mathrm{e}} \sim 7.5 \times 10^{3}$ at the East points and $N_{\mathrm{e}} \sim 3 \times 10^{3}$ at the West ones, we obtain: $T_{\mathrm{e}} \approx 8000 \mathrm{~K}$ at the center, $T_{\mathrm{e}} \approx 8100 \mathrm{~K}$ at the East points and $T_{\mathrm{e}} \approx 6800 \mathrm{~K}$ at the West points respectively. Some authors supposed a similar, but radial, gradient (Wilson \& Jaeger 1987; Subramanyan et al. 1988), while others did not (Wilson et al. 1997). This observed tendency could be due to a corresponding density gradient (Wilson \& Jaeger 1987). So far we have been unable to find a satisfactory expanation for it.

\section{Summary}

Results of a common observing program between Italy $(66 \alpha)$ and Russia ( $56 \alpha$ ) have been presented, and can be sumarized as follow:

- The distribution of the ionized helium abundance $\left(y^{+}=\right.$ $\left.N\left(\mathrm{He}^{+}\right) / N\left(\mathrm{H}^{+}\right)\right)$over the Orion A nebula has been obtained. Such a distribution explains the discrepancy present in previous observations. The present results confirm that the $\mathrm{He}^{+}$zone is smaller than the $\mathrm{H}^{+}$zone, with a different ratio of their core and envelope sizes and that it depends on the direction from the HII center;

- The obtained helium abundance $y=10.0( \pm 0.8) \%$ is in agreement with optical values when the correction for the 
ionization structure has been made. The estimate of the primordial helium abundance by mass is $Y_{\mathrm{p}}=(25.2 \pm 1.8) \%$;

- The behaviour of both radial velocities and widths of RRLs supports the asymmetric "blister-type" structure of the HII region, which expands asymmetrically along the main axis relative to the molecular cloud, mainly toward the observer;

- The LTE electron temperatures have been derived for the first 9 positions, and are found in the range from $\sim 7800 \mathrm{~K}$ to $\sim 9600 \mathrm{~K}$. An interesting trend is observed: the West points are at lower temperatures than the East ones.

This high sensitivity mapping require long integration times, not possible in a short observing period. For a clearer picture, further observations are needed, especially for the positions of Ring-3 and partly, for Ring-2.

Acknowledgements. This work was partially supported by the Russian State contract 40.022.1.1.1106 ("Cosmion" Project), by the Italian CNR and Russian Academy of Science Agreement, ASI and MURST. A.P.T. particularly acknowledges the Italian CNR for support during his visits to Bologna. The authors wish also to thank the anonymous referee for helpful comments and suggestions. Based on observations with the Medicina telescope operated by INAF - Istituto di Radioastronomia.

\section{References}

Aannestad, P. A. 1989, ApJ, 338, 162

Adler, D. S., Wood, D. O. S., \& Goss, W. M. 1996, ApJ, 471, 871

Baldwin, J. A., Ferland, G. J., Martin, P. G., et al. 1991, ApJ, 374, 580

Balick, B., Gammon, R. H., \& Hjellming, R. H. 1974, PASP, 86, 616

Berulis, I. I., Gusev, V. A., Kutsenko, A. A., et al. 1983, Trudy Fiz. Inst. Lebedeva Akad. Nauk SSR, 135, 35

Bodenheimer, P., Tenorio-Tagle, G., \& Yorke, H. W. 1979, ApJ, 233, 85

Churchwell, E., Mezger, P. C., \& Huchtmeier, W. 1974, A\&A, 32, 283

Comoretto, G., \& Palagi, F. 1993, Arcetri Technical Report, 13, 93

Dent, W. A. 1972, ApJ, 177,93

Ershov, D., Gulyaev, S. A., Ivanchik, A., Varshalovich, A. D., \& Tsivilev, A. P. 1998, A\&AT, 15, 281

Esteban, C., Peimbert, M., Torres-Peimbert, S., \& Escalante, V. 1998, MNRAS, 295,401
Gordon, M. A. 1989, ApJ, 337, 782

Gordon, M. A., \& Sorochenko, R. L. 2002, Radio Recombination Lines. Their Physics and Astronomical Applications, Astrophysics and Space Science Library (Dordrecht: Kluwer Academic Publishers), Vol. 282

Gulyaev, S. A., Sorochenko, R. L., \& Tsivilev, A. P. 1997, Astron. Lett., 23, 165 Hua, C. T., \& Louise, R. 1982, Ap\&SS, 88, 477

Izotov, Yu. I., \& Thuan, Tr. X. 2004, ApJ, 602, 200

Jaffe, D. T., \& Pankonin, V. 1978, ApJ, 226, 869

Lockman, F. L., \& Brown, R. L. 1982, ApJ, 259, 595

Pagel, B. E. J. 2000, Physics Rep., 333, 433

Pankonin, V., Walmsley, C. M., \& Harwit, M. 1979, A\&A, 75, 34

Pankonin, V., Walmsley, C. M., \& Thum, C. 1980, A\&A, 89, 173

Peimbert, M., \& Torres-Peimbert, S. 1977, MNRAS, 179, 217

Peimbert, M., Ukita, N., \& Hasegava, T. 1988, PASJ, 40, 581

Ponomarev, V. O. 1996, Preprint FIRAN, N 54, Moscow

Roelfsema, P. R., Goss, W. M., Whiteoek, J. B., Gardner, F. F., \& Pankonin, V. 1987, A\&A, 175, 219

Roelfsema, P. R., Goss, W. M., \& Malnik, D. C. 1992, ApJ, 394, 188

Rubin, R. H. 1984, ApJ, 287, 653

Rubin, R. H., Simpson, J. P., Hass, M. R., \& Erickson E. F. 1991, ApJ, 374, 564

Salem, M., \& Brocklehurst, M. 1979, ApJSS, 39, 633

Schmid-Burgk, J. 1981, Nuclear Astrophysics. Proc. Int. School of Nuclear

Physics, ed. D. H. Wilkinson (Oxford: Pergamon Press), 6, 295

Shaver, P. A. 1980, A\&A, 90, 34

Shaver, P. A., McGee, R. X., Newton, L. M., Danks, A. C., \& Pottasch, S. R. 1983, MNRAS, 204, 53

Smirnov, G. T., \& Tsivilev, A. P. 1982, Sov. Astron. Lett., 26, 616

Sorochenko, R. L., \& Tsivilev, A. P. 1996, Proc. of 1 Int. conf. on cosmoparticle physics, Cosmion-94, held in Moscow 1994, ed. M. Yu. Khlopov, M. E. Prokhorov, A. A. Starobinsky, \& J. Tran Thanh Van (Éditions Frontières), Cosmoparticle physics, 1,123

Sorochenko, R. L., \& Tsivilev, A. P. 2000, Astron. Rep., 44, 426

Subramanyan Gopal-Krishna, R., et al. 1988, VA, 31, 207

Tsivilev, A. P. 1991, Sov. Astron. Lett., 17, 1

Tsivilev, A. P., Ershov, A. A., Smirnov, G. T., \& Sorochenko, R. L. 1986, Sov. Astron. Lett., 12, 355

Walker, T. P., Steigman, G., Schramm, D. N., Olive, K. A., \& Kang Ho-Shik 1991, ApJ, 376, 51

Wilson, T. L., \& Jaeger, B. 1987, A\&A, 184, 291

Wilson, T. L., \& Pauls, T. 1984, A\&A, 138, 225

Wilson, T. L., Filges, L., Codella, C., Reich, W., \& Reich, P. 1997, A\&A, 327, 1177

Wyrowski, F., Schilke, P., Hofner, P., \& Walmsley, C. M. 1997, ApJ, 487, L171

Zel'dovich, B. Ya., \& Novikov, N. D. 1975, Structure and evolution of Universe,

Moscow, Nauka

Zuckerman, B. 1973, ApJ, 183, 863 
S. Poppi et al.: Helium abundance and ionization structure in the Orion nebula, Online Material $p 1$

\section{Online Material}




\subsection{Carbon RRLs}

To obtain a genuine detection, therefore, one needs to separate the helium from the carbon profiles. That has been possible only for the $66 \alpha$ spectrum. For the $56 \alpha$ line the frequency resolution was not enough fine to distinguish the carbon lines, which made the helium profile asymetric and shifted it to higher frequency. On the other hand, in previous $125 \mathrm{kHz}$ frequency resolution observations, the RRL C56 $\alpha$ was clearly detected in both Ori Bar (Sorochenko \& Tsivilev 2000) with RT22.

To separate the $C 56 \alpha$ profile from the helium profile, we reduce the number of free parameters for the carbon profiles, by fixing the width and performing several fits, varying it whithin the interval 2-6 $\mathrm{km} \mathrm{s}^{-1}$, and looking for minimization of the $\chi^{2}$ value. This range has been chosen on the basis of other observations, including the $66 \alpha$ presented here. For example, in the Ori Bar position (and similarly for the Ori A) we obtained $\Delta V=$ $3.4( \pm 0.9) \mathrm{km} \mathrm{s}^{-1}$ for the $C 56 \alpha$ line (Sorochenko \& Tsivilev 2000); for the $C 91 \alpha$, line, $\Delta V$ was between 2 and $2.5 \mathrm{~km} \mathrm{~s}^{-1}$ (Wyrowski et al. 1997). However, $30 \%$ of the carbon intensity has been lost in the data reduction since the real $\Delta V$ could range from 2.5 to $3.3 \mathrm{~km} \mathrm{~s}^{-1}$.

Thus, the radio recombination lines of carbon $(C 56 \alpha$ and $C 66 \alpha$ ) were seen in most of the sample. Their radial velocities $\left(V_{\mathrm{LSR}}\right)$ lay in the interval from +8 to $+11 \mathrm{~km} \mathrm{~s}^{-1}$, in agreement with the previous carbon line investigation of Jaffe \& Pankonin (1978), while $H$ and He RRLs have $V_{\text {LSR values rang- }}$ ing from +1 to $-6 \mathrm{~km} \mathrm{~s}^{-1}$. Since carbon velocities refer mainly to molecular cloud motion while the hydrogen ones refer to the motion of ionized material, their difference shows again the HII region asymmetric expansion toward the observer, relative to the associated molecular cloud.

On the other hand, carbon radial velocities have a more positive trend than the lines from the core of the molecular cloud (e.g. $V_{\mathrm{LSR}} \approx 8 \mathrm{~km} \mathrm{~s}^{-1}$ of OCS line detected here, Fig. 2; see also Balick et al. 1974) and this means that the background CII region, as a HI-HII interface, is moving slightly into the molecular cloud (see e.g. Balick et al. 1974; and Jaffe \& Pankonin 1978). 
S. Poppi et al.: Helium abundance and ionization structure in the Orion nebula, Online Material p 3

\section{Appendix: Spectra plots}
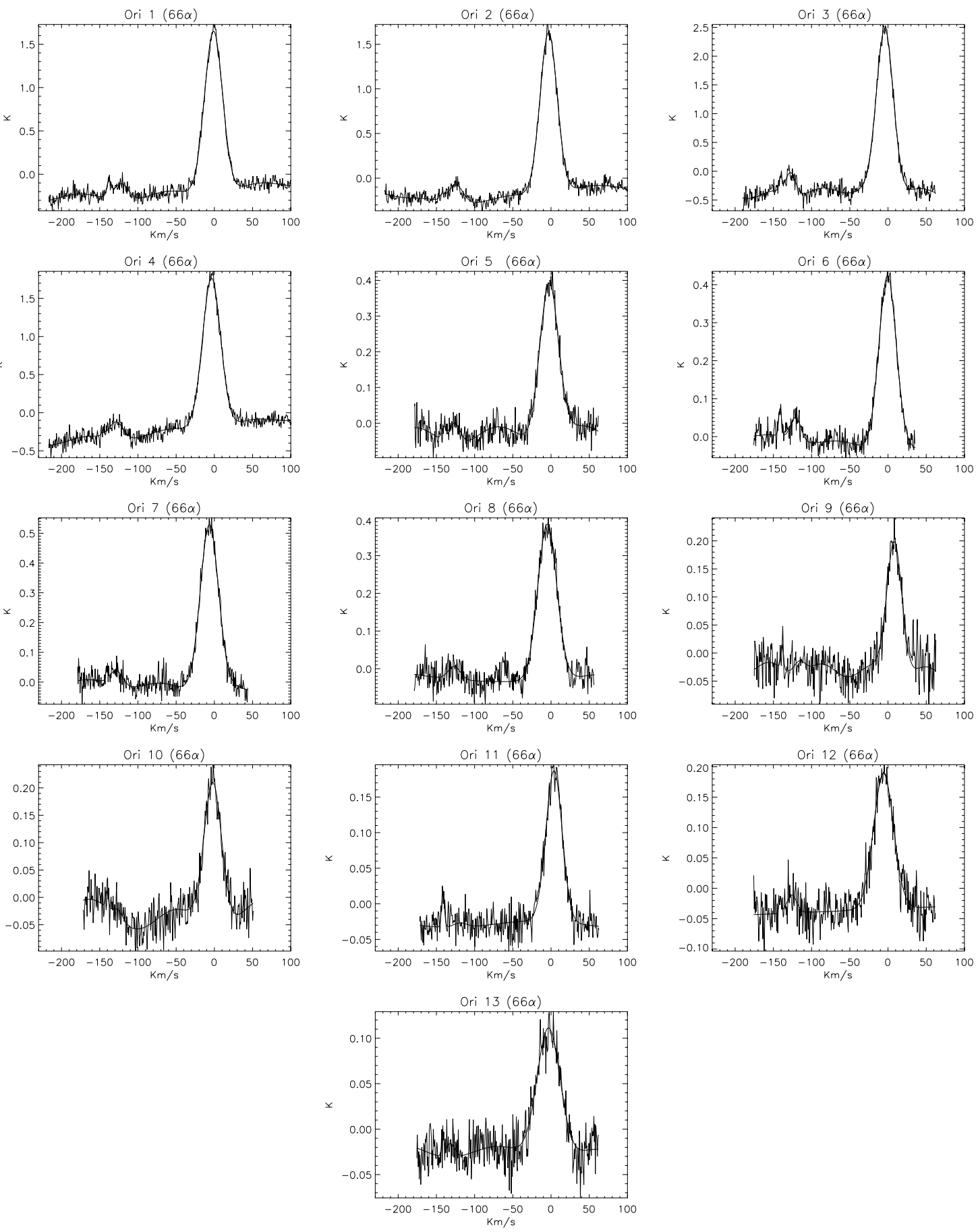

Fig. 15. Obtained $66 \alpha$ spectra. $Y$-axis scale is in antenna temperatures $(\mathrm{K})$, the $x$-axis scale is in velocity terms, where: $V_{\mathrm{LSR}} \approx 0 \mathrm{~km} \mathrm{~s}^{-1}-$ is the hydrogen line position $(\mathrm{H}), V_{\mathrm{LSR}} \approx-122 \mathrm{~km} \mathrm{~s}^{-1}$ is the helium line position $(\mathrm{He}), V_{\mathrm{LSR}} \approx-140 \mathrm{~km} \mathrm{~s}^{-1}$ is the carbon line position (C). 
S. Poppi et al.: Helium abundance and ionization structure in the Orion nebula, Online Material $p 4$
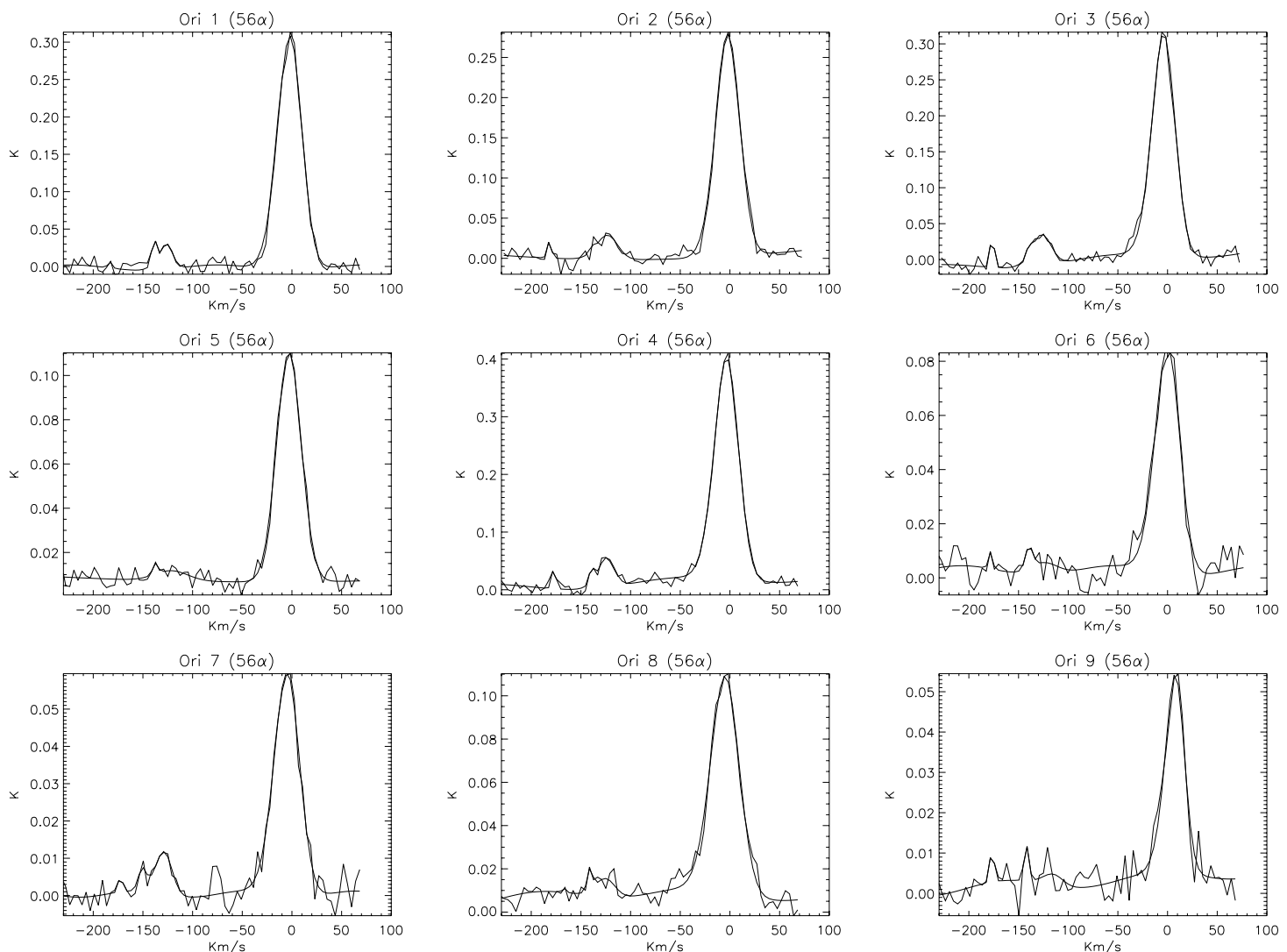

Fig. 16. Obtained $56 \alpha$ spectra. $Y$-axis scale is in antenna temperatures $(\mathrm{K})$, the $x$-axis scale is in velocity terms, where: $V_{\mathrm{LSR}} \approx 0 \mathrm{~km} \mathrm{~s}{ }^{-1}-$ is the hydrogen line position $(\mathrm{H}), V_{\mathrm{LSR}} \approx-122 \mathrm{~km} \mathrm{~s}^{-1}$ is the helium line position $(\mathrm{He}), V_{\mathrm{LSR}} \approx-140 \mathrm{~km} \mathrm{~s}^{-1}$ is the carbon line position $(\mathrm{C})$ and $V_{\mathrm{LSR}} \approx-180 \mathrm{~km} \mathrm{~s}^{-1}$ is the molecular line position, OCS. 